\title{
Tuning the Continuum of Structural States in the Native Ensemble of a Regulatory Protein
}

\author{
Abhishek Narayan and Athi N. Naganathan*(i) \\ Department of Biotechnology, Bhupat \& Jyoti Mehta School of Biosciences, Indian Institute of Technology Madras, Chennai 600036, \\ India
}

Supporting Information

ABSTRACT: The mesoscale nature of proteins allows for an efficient coupling between environmental cues and conformational changes, enabling their function as molecular transducers. Delineating the precise structural origins of such a connection and the expected spectroscopic response has, however, been challenging. In this work, we perform a combination of urea-temperature double perturbation experiments and theoretical modeling to probe the conformational landscape of $\mathrm{Cnu}$, a natural thermosensor protein. We observe unique ensemble signatures that point to a continuum of conformational substates in the native ensemble and that respond intricately to perturbations upon monitoring secondary and tertiary structures, distances between an intrinsic FRET pair, and hydrodynamic volumes. Binding assays further reveal a weakening of the Cnu functional complex with temperature, highlighting the molecular origins of signal transduction critical for pathogenic response in enterobacteriaceae.
$\mathrm{I}^{\mathrm{t}}$ $t$ is well established that proteins sample a variety of functionally relevant conformations in their native ensemble. ${ }^{1,2}$ The flexibility arises from the weak noncovalent nature of the stabilizing interactions, the large degree of freedom associated with the main chain, and the finite sizes of protein molecules. The resultant mesoscopic nature of proteins translates to large surface-area-to-volume ratios, thus contributing to specific interactions with the solvent molecules and large solvent-coupled fluctuations even when in thermodynamic equilibrium. 3 Solvent properties can, therefore, be tuned either by temperature or by adding cosolvents (urea or guanidinium hydrochloride) to modulate these interactions and hence perturb the folding landscape of proteins. Perturbation experiments have therefore contributed immensely to the understanding of protein properties, particularly in two-state-like systems. ${ }^{5,6}$ In such proteins, adding cosolvents tunes the relative macroscopic populations of the folded and unfolded states, resulting in distinct sigmoidal-like unfolding curves. However, it has generally been challenging to extract or interpret the origin of signals beyond a simple two-state equilibrium because of the complexity intrinsic to such analysis. ${ }^{7}$ In fact, double-perturbation experiments involving cosolvents and temperature reveal distinct signal dependencies in globally downhill and incipient downhill folders, ${ }^{8,9}$ arising from the differences in the structural features of ensembles that are populated in response to one perturbation and that are tuned by another. Such an intrinsically tunable landscape allows for proteins to act as molecular transducers or rheostats; that is, they couple the changes in ambient conditions to their conformations that in turn can determine the functional response. $^{10}$

In this regard, it was recently identified that the four-helix bundle protein $\mathrm{Cnu}$ (Figure 1a), a single gene product, displays thermosensor-like properties that are critical for efficient pathogenic response in enterobacteriaceae that commonly infect human gastrointestinal tracts. ${ }^{11}$ Global spectroscopic, site-specific NMR experiments, hydrodynamic measurements, theoretical modeling, and simulations indicate that the $\mathrm{Cnu}$ native ensemble is best described by an array of conformational states that are in dynamic equilibrium with one another in a single broad native well. If this is indeed the case, then solvent modulations with chemical denaturants together with thermal perturbations should result in nontrivial effects on the folding landscape. Moreover, the cosolvent- or temperature-dependent spectroscopic signatures are expected to be different from conventional observations. ${ }^{5}$ To explore these issues in detail, we monitor the response of the native ensemble of $\mathrm{Cnu}$ to perturbations by urea and temperature with far- and near-UV circular dichroism (CD), fluorescence (specifically, tyrosinetryptophan resonance energy transfer), hydrodynamic measurements, simulations and also perform binding studies.

We first probe the features of Cnu folding landscape with a variant of the statistical mechanical Wako-Saitô-MuñozEaton (WSME) model. ${ }^{12-14}$ Using identical parameters as a previous study, a chemical denaturant dependence is introduced following the linear free-energy relation commonly observed in experiments ${ }^{15}$ (see the Supporting Information) and as employed before. ${ }^{16}$ Such a perturbation reveals that the native ensemble of $\mathrm{Cnu}$ can be coarsely divided into two subensembles, $\mathrm{N}$ and $\mathrm{N}^{*}$ (Figure $1 \mathrm{~b}$ ). Importantly, their properties vary as a function of both temperature and cosolvent

Received: February 27, 2017

Accepted: March 27, 2017

Published: March 27, 2017 

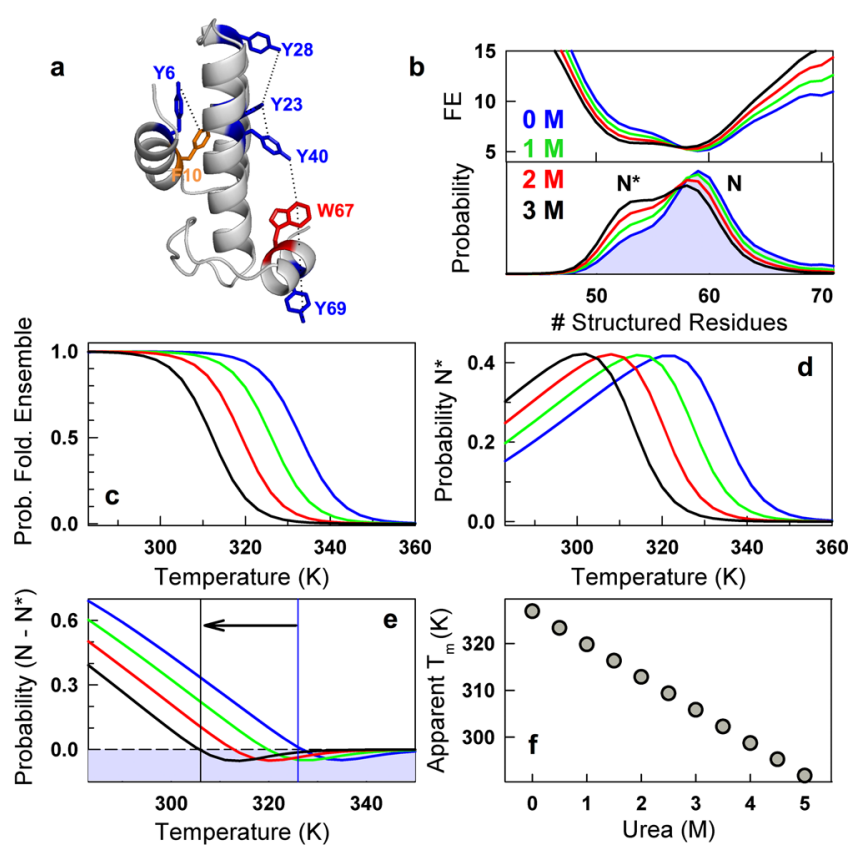

Figure 1. WSME model predictions. (a) Structure of Cnu highlighting the various aromatic residues. Note that W67 is in the fourth helix while Y40 is in the third helix. (b) Expected changes in the 1D freeenergy profile (in $\mathrm{kJ} \mathrm{mol}^{-1}$ ) and the corresponding populations as a function of urea at $278 \mathrm{~K}$. $(\mathrm{c}-\mathrm{e})$ Predicted changes in the population of the folded ensemble, $\mathrm{N}^{*}$, and the relative population as a function of temperature at various urea concentrations. (f) Apparent melting temperature at different urea concentrations measured as the temperature at which $\mathrm{N}^{*}$ starts to dominate over $\mathrm{N}$ (arrow in panel e).

concentration. This can be seen as horizontal shifts in the positions of the population maxima or free-energy minima of both $\mathrm{N}$ and $\mathrm{N}^{*}$ toward more disorder (Figure $1 \mathrm{~b}$ ). This suggests that probes that are sensitive to the fine features of the landscape should reveal distinct spectroscopic signals under each of the conditions. In addition to this, the populations of the native ensemble, $\mathrm{N}^{*}$, and the relative difference in population between $\mathrm{N}$ and $\mathrm{N}^{*}$ are predicted to result in sigmoidal, broad and near-parabolic, and linearly decreasing temperature dependencies at individual cosolvent concentrations, respectively (Figure $1 \mathrm{c}-\mathrm{e}$ ). The resulting apparent $T_{\mathrm{m}}$, measured as the temperature at which the signals crossover in signs, should follow a linear trend with urea (Figure 1f).

Cnu has one tryptophan in the fourth helix and five tyrosines that are distributed throughout the structure. In proteins rich in aromatic residues, near-UV CD spectral analysis can provide detailed structural information, as they are sensitive to the tertiary packing environment of tyrosine and tryptophan. ${ }^{17}$ The near-UV CD spectral signatures of $\mathrm{Cnu}$ at four different urea concentrations ( 0 to $3 \mathrm{M}$ ) reveal distinct amplitudes for the overall- and relative-spectral bands $(265,270,280$, and 290 $\mathrm{nm}$ ), clearly indicating that distinct ensembles are populated at these urea concentrations (for example, compare the spectra at 1 and $3 \mathrm{M}$ urea in Figure 2a). Remarkably, the signals at 290 and $280 \mathrm{~nm}$ follow the exact same trend predicted by the WSME model, suggesting that they probe the overall population of the native ensemble and that of $\mathrm{N}^{*}$, respectively (Figure 2b,c). SVD (singular-value decomposition) analysis of the temperature-wavelength spectra reveals an anticorrelation between the bands of tyrosine and tryptophan in the second component that reports on spectral changes (Figure $2 \mathrm{~d}$ ). The
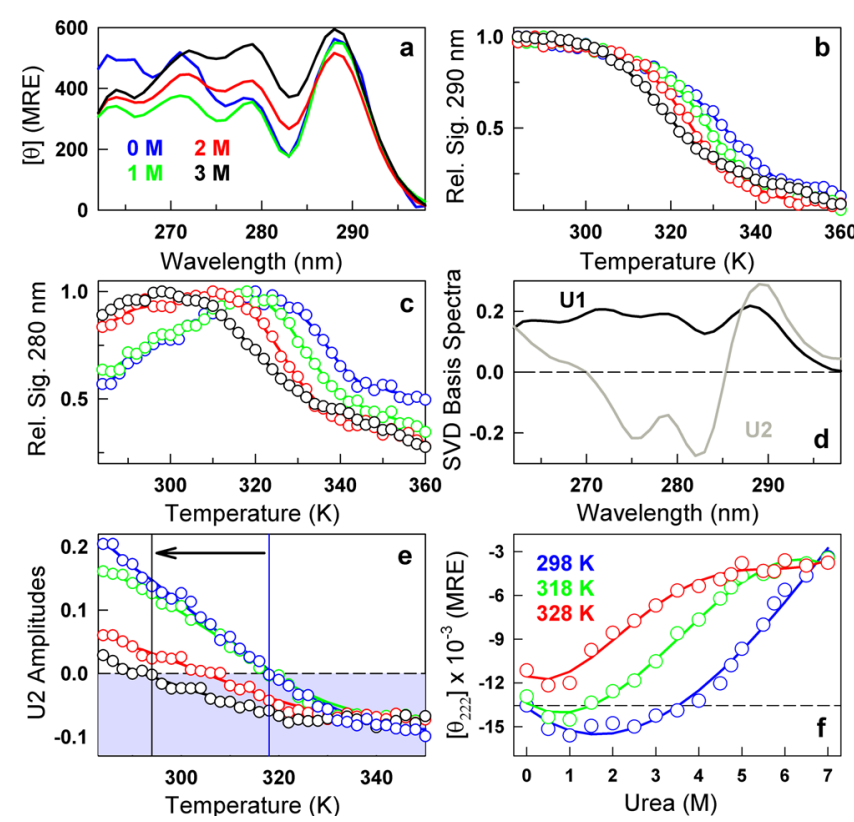

Figure 2. Unique solvent sensitivity of Cnu. Panels a-e follow the same color code as panel a. Lines in panels $b-f$ are shown to guide the eye. $(\mathrm{a}-\mathrm{c})$ Near-UV CD spectra at $298 \mathrm{~K}$ in mean residue ellipticity (MRE) units of deg $\mathrm{cm}^{2} \mathrm{dmol}^{-1}$ and normalized unfolding curves at 290 and $280 \mathrm{~nm}$ at different urea concentrations. (d) Spectral signatures of the two significant components from an SVD of the urea-temperature near-UV CD data. (e) Amplitude of the second component as a function of temperature and urea. Note that the temperatures at which the amplitudes change sign (vertical lines) are urea-dependent (arrow in panel e). (f) Far-UV CD monitored changes in secondary structure as a function of urea at representative temperatures. The dashed line signals the molar ellipticity at $0 \mathrm{M}$ and $278 \mathrm{~K}$

amplitude of this component decreases linearly with temperature and changes sign (positive to negative) at specific temperatures as a function of urea (Figure 2e). This observation is also in accordance with the predictions of the WSME model that points to this dependence to be originating from the differences in the populations of $\mathrm{N}$ and $\mathrm{N}^{*}$ (Figure $1 \mathrm{e})$.

The urea-dependent far-UV CD signal at $222 \mathrm{~nm}$ again displays a pattern that has not been reported in any protein system: the signal intensity increases (becomes more negative) with the urea concentration, reaches a plateau, and then decreases in intensity in a sigmoidal fashion (Figure $2 \mathrm{f}$ and Supporting Information Figure S1). The position of the minima moves toward lower urea concentrations and concomitantly decreases in magnitude, suggestive of a malleable native ensemble. What could contribute to this unique dependence? Careful analysis of the spectral features of far-UV CD bands in proteins has shown that tyrosine exhibits a strong positive band when in a helical conformation. ${ }^{18}$ The fact that $\mathrm{Cnu}$ has five tyrosines and that the signal intensity increases with urea suggests that some tyrosines populate nonhelical conformations even at $298 \mathrm{~K}$, despite the overall structure appearing to be folded. As the temperature is increased, the probability of the unfolded ensemble increases, thus resulting in a decrease in the signal intensity. In other words, the observed rollover in far-UV CD signals at $222 \mathrm{~nm}$ arises from a delicate balance between these two features. 
Circular dichroism experiments highlight the native ensemble of $\mathrm{Cnu}$ to be changing both its secondary and tertiary structure with solvent perturbations in a distinct manner. The structural changes are more probable at the C-terminal helix due to its weak packing and the large conformational flexibility of the loop connecting the third and fourth helices. ${ }^{11} \mathrm{We}$ therefore expect the relative distances between W67 (located in the fourth helix) and Y40 (in the third helix; Figure 1a) to increase with perturbation magnitude within the native ensemble, that is, in the pretransition region where there is only a minimal population of the unfolded state.

The quantum yield (QY), as estimated by exciting the protein at $274 \mathrm{~nm}$, increases with urea concentration, reaches a plateau, and then decreases sigmoidally, mirroring far-UV CD observations (Figure 3a). The apparent chemical midpoint is
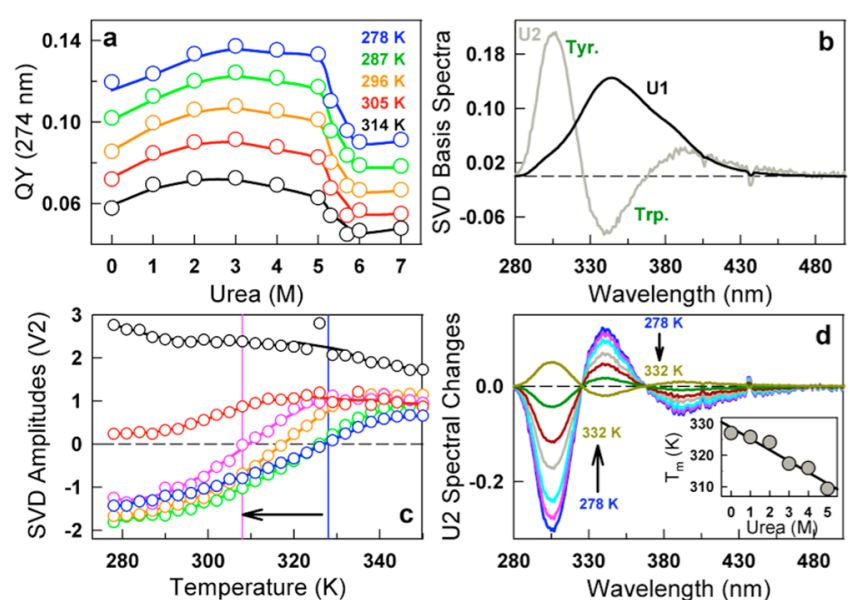

Figure 3. Fluorescence monitored structural changes in the native ensemble. Lines in panels $a$ and $c$ are shown to guide the eye. (a) Quantum yield (QY) of the protein upon excitation at $274 \mathrm{~nm}$ as a function of urea at select temperatures. (b) Spectral signatures of the two significant components, U1 and U2, from SVD of the ureatemperature fluorescence data. (c) Temperature dependence of the amplitude of the second component at select urea concentrations of 0 (blue), 1 (green), 3 (orange), 5 (magenta), 5.3 (red), and $6 \mathrm{M}$ (black). Note that the temperatures at which the amplitudes change sign (vertical lines) are urea-dependent (arrow in panel c). (d) Effective spectral change at $0 \mathrm{M}$ urea, obtained by multiplying $\mathrm{U} 2$ with $\mathrm{V} 2$, is shown as a representative example to highlight the changes. Inset plots the temperatures at which the signs change at different urea concentrations (from panel c).

estimated to be $\sim 5.3 \mathrm{M}$ at $298 \mathrm{~K}$ from a first-derivative analysis of the QY data. To understand the possible structural changes that contribute to this unique observation, we perform a global SVD of the raw temperature/urea-wavelength fluorescence data. The spectral deconvolution results in two significant components, the first of which represents the average spectrum, and its amplitude accounts for the intrinsic temperature dependence of fluorescence (Figure S2). The second SVD component again points to an anticorrelation similar to that observed in near-UV CD spectral analysis but this time between the emission bands of tyrosine (emission maximum $\sim 305 \mathrm{~nm}$ ) and tryptophan (emission maximum $\sim 340 \mathrm{~nm}$; Figure $3 \mathrm{~b}$ ). However, unlike near-UV CD signals that are challenging to interpret, the changes in fluorescence intensities have a clear structural origin. Specifically, there can be a FRET (Förster resonance energy transfer) between tyrosine (donor) and tryptophan (acceptor) if they are close in space. In fact, the
$\mathrm{C}_{\alpha}-\mathrm{C}_{\alpha}$ distance between $\mathrm{W} 67$ and $\mathrm{Y} 40$ is $\sim 11 \AA$ in the native structure, very close to the expected $R_{0}$ for this FRET pair ( 9-12 $\AA$ ). The FRET-induced effects can be more clearly seen in the plot of the amplitudes of the second component that changes continually with increasing temperature and changes sign at specific temperatures depending on the urea concentration (Figure 3c). In other words, the low tyrosinetryptophan distance between W67 and Y40, as expected of a fully folded structure at lower temperatures, results in a larger FRET between this pair and hence a decreased intensity for tyrosine band (negative spectrum) and an increased intensity for tryptophan band (positive spectrum) at $278 \mathrm{~K}$ (Figure 3d, obtained by multiplying U2 with V2). At higher temperatures, the distance between W67 and Y40 increases due to partial unfolding; this results in reduced FRET between this pair and hence the tyrosine spectrum dominates over that of tryptophan (Figure 3d). The relative dominance of tyrosine emission over tryptophan depends on the urea concentration, and this results in a linear decrease in apparent melting temperatures with urea (inset to Figure 3d), consistent with predictions from the WSME model (Figure 1f). Interestingly, we also find evidence for temperature-induced collapse in the unfolded ensemble using this simple technique, very similar to other FRET-based observations. ${ }^{19}$ This can be seen as a decrease in FRET intensity (Y40-W67 getting closer) with increasing temperatures at $6 \mathrm{M}$ urea (black in Figure 3c).

The continuous increase in the W67-Y40 distances as a function of temperature or urea in the native ensemble should result in a concomitant increase in the hydrodynamic volumes. We clearly observe an increase in the Stokes radius of the protein with increasing urea concentration at $278 \mathrm{~K}$ from analytical size-exclusion chromatography (vertical bars in Figure 4a). The protein dimensions approach that of a molten-globule at $278 \mathrm{~K}$ and $3 \mathrm{M}$ urea and $298 \mathrm{~K}$ and $0 \mathrm{M}$ urea (continuous line in Figure 4a) following the empirical formula of Uversky and coworkers ${ }^{20}$ (dashed line in Figure 4a). These observations highlight that fixing urea concentration and tuning temperature or vice versa will result in an equivalent effect on the native ensemble. This reciprocity is particularly advantageous because it is relatively easier to simulate temperature effects and importantly perform binding experiments where temperature is modulated.

We therefore supplement the previous $6 \mu \mathrm{s}$ of $\mathrm{MD}$ simulations in explicit water $(280 \text { and } 310 \mathrm{~K})^{11}$ with another $3 \mu \mathrm{s}$ of cumulative simulation time at $295 \mathrm{~K}$. The resulting $\mathrm{C}_{\alpha}-$ $\mathrm{C}_{\alpha}$ distance distribution between the Y40-W67 pair is nearunimodal at $280 \mathrm{~K}$, indicative of a well-folded ensemble (blue in Figure 4b). Despite the limited sampling, increasing the temperature perturbs the native ensemble dramatically with the Y40-W67 distances spanning a large range with distinct conformational substates (Figure $4 \mathrm{~b}$ ) exactly as expected from the experimental FRET temperature dependence. Coarsegrained simulations also point to a similar feature in the native ensemble (Figure S3). The consistency between the three approaches employed here-experiments, theoretical modeling, simulations-provides strong evidence that the native ensemble of $\mathrm{Cnu}$ is a structural continuum.

The sensitivity of Cnu to solvent conditions, a feature expected of protein molecular rheostats, raises questions on the biological necessity for a tunable native ensemble. ${ }^{21,22}$ Because the enterobacteriaceae family predominantly infects human hosts, the constant body temperature of $310 \mathrm{~K}$ becomes a major thermodynamic variable. Microbiological-biochemical 

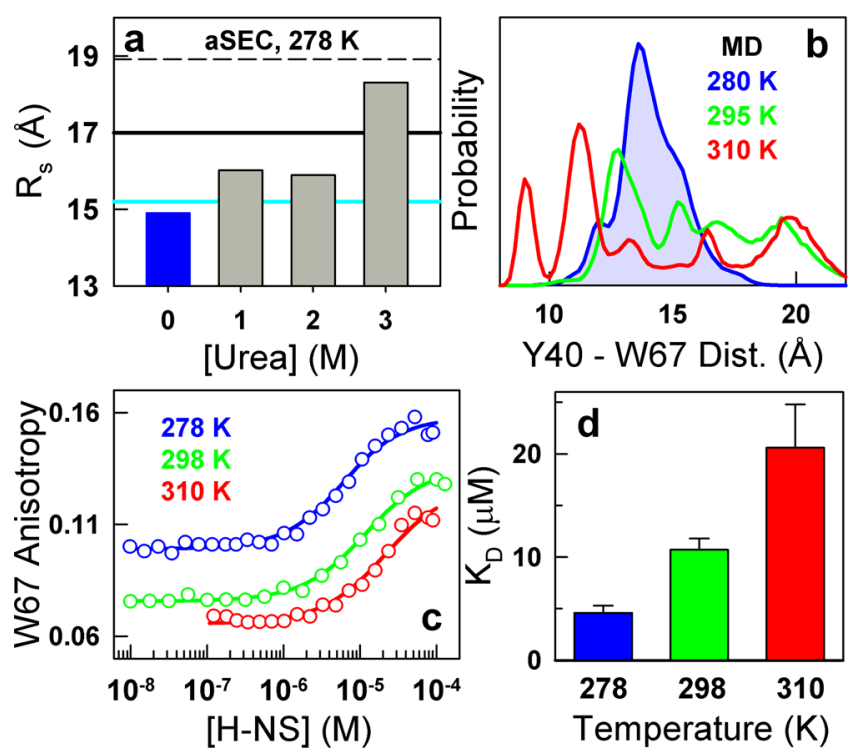

Figure 4. Structural swelling and functional significance. (a) Changes in the Stokes radius as monitored by analytical size-exclusion chromatography (aSEC) at $278 \mathrm{~K}$ and at various urea concentrations ( $0 \mathrm{M}$ is in blue while the rest are in gray bars). The horizontal cyan and black lines signal the protein dimensions as measured by analytical ultra centrifugation at 278 and $298 \mathrm{~K}$, respectively. The horizontal dashed line indicates the expected dimension of a molten-globular conformation of Cnu from size-scaling arguments. (b) Distribution of $\mathrm{C}_{\alpha}-\mathrm{C}_{\alpha}$ distances between Y40 in the third helix and W67 in the fourth helix from all-atom MD simulations at different temperatures. (c) Changes in tryptophan anisotropy upon titration with $\mathrm{H}_{-15} \mathrm{NS}_{1-59}$ at select temperatures. (d) Apparent dissociation constants from fits (line in panel c) assuming a 1:1 binding equilibrium.

experiments have also shown that a complex between Cnu and $\mathrm{H}-\mathrm{NS}$ represses pathogenic response at low temperatures $(\sim 280 \mathrm{~K})$ while promoting the expression of toxins at higher temperatures ( $\sim 310 \mathrm{~K}$; the body temperature of humans). ${ }^{23} \mathrm{It}$ is the molecular patch formed by the helices 3 and 4 of $\mathrm{Cnu}$ that is responsible for binding with H-NS. ${ }^{24,25}$ Because experiments and simulations point to a continuous increase in the distances between these two helices with changing solvent conditions, it points to a simple mechanism by which the binding affinity can be regulated: the binding interface should be well formed at $280 \mathrm{~K}$, thus promoting complex formation while the interface should be destabilized at high temperatures and thus disfavoring complex formation (Figure $\mathrm{S} 3 \mathrm{~b})$. To test for this experimentally, we monitored the change in tryptophan fluorescence anisotropy of $\mathrm{Cnu}$ by titrating it with $\mathrm{H}-\mathrm{NS}_{1-59}$ at different temperatures (Figure $4 \mathrm{c}$ ). We find that the binding affinity decreases by a factor of $\sim 5$ between 278 and $310 \mathrm{~K}$ (Figure 4d). However, our interpretation is complicated by the fact that $\mathrm{H}_{-} \mathrm{NS}_{1-59}$, a fragment of a larger protein, by itself displays a steep pretransition upon thermal modulations (Figure S4), indicating that it also undergoes structural loss in the same temperature range. While it is not clear if the full-length H-NS would undergo a similar structural change, our work provides first experimental evidence that a synergy between the tunable conformational ensembles of both the proteins potentially dictates the extent of pathogenic response.

Our results show that the structure of protein $\mathrm{Cnu}$ is highly malleable, displaying hitherto undocumented complexity and thus making it optimally sensitive to fluctuations in the environment. Such a feature has been theoretically predicted before for even folded single-domain proteins ${ }^{26,27}$ and is increasingly being observed by several different experimental approaches including ensemble multisite FRET, ${ }^{28}$ singlemolecule FRET, ${ }^{29}$ calorimetric measurements, and $\mathrm{NMR}^{30}$ These observations including ours also highlight that ensemble descriptions, which account for the statistical nature of protein chains, are the way forward, and such an approach can provide a reliable basis for deciphering complex spectroscopic signals. Because natural selection acts at the level of function, the inherently tunable nature of proteins should have a precise functional reason that we exemplify here using Cnu as a model system. Bacteria owe their survival in extremes of environmental conditions to molecular sensors like Cnu. It is therefore natural to expect the possibility of similar coupling between the conformational features of the folding landscape of several other proteins to extrinsic variables like $\mathrm{pH}$ and ionic strength. Understanding the molecular origins of environmental sensitivity from such natural sensors could pave the way for effective protein design strategies and potentially reveal promising drug targets.

\section{ASSOCIATED CONTENT}

\section{Supporting Information}

The Supporting Information is available free of charge on the ACS Publications website at DOI: 10.1021/acs.jpclett.7b00475.

Methods, the far-UV CD double-perturbation data, amplitudes of the fluorescence second component compared against experimental QY measures, Y40W67 distances from coarse-grained simulations, and the unfolding curve of $\mathrm{H}_{-} \mathrm{NS}_{1-59}$. (PDF)

\section{AUTHOR INFORMATION}

\section{Corresponding Author}

*E-mail: athi@iitm.ac.in.

ORCID $\odot$

Athi N. Naganathan: 0000-0002-1655-7802

Notes

The authors declare no competing financial interest.

\section{ACKNOWLEDGMENTS}

This work was funded by the grants BT/06/IYBA/2012-14 from the Department of Biotechnology (DBT) and IA/I/15/1/ 501837 from the Wellcome Trust/DBT India Alliance Intermediate Fellowship to A.N.N. We acknowledge the FIST facility sponsored by the Department of Science and Technology (DST), India at the IITM.

\section{REFERENCES}

(1) Bryngelson, J. D.; Onuchic, J. N.; Socci, N. D.; Wolynes, P. G. Funnels, Pathways, and the Energy Landscape of Protein-Folding - a Synthesis. Proteins: Struct., Funct., Genet. 1995, 21, 167-195.

(2) Boehr, D. D.; Nussinov, R.; Wright, P. E. The role of dynamic conformational ensembles in biomolecular recognition. Nat. Chem. Biol. 2009, 5, 789-796.

(3) Frauenfelder, H.; Fenimore, P. W.; Young, R. D. Protein dynamics and function: insights from the energy landscape and solvent slaving. IUBMB Life 2007, 59, 506-512.

(4) Fenimore, P. W.; Frauenfelder, H.; McMahon, B. H.; Parak, F. G. Slaving: solvent fluctuations dominate protein dynamics and functions. Proc. Natl. Acad. Sci. U. S. A. 2002, 99, 16047-16051.

(5) Tanford, C. Protein denaturation. Adv. Protein Chem. 1968, 23, 121-282. 
(6) Schellman, J. A. The Thermodynamics of Solvent Exchange. Biopolymers 1994, 34, 1015-1026.

(7) Tanford, C. Protein denaturation. C. Theoretical models for the mechanism of denaturation. Adv. Protein Chem. 1970, 24, 1-95.

(8) Oliva, F. Y.; Muñoz, V. A simple thermodynamic test to discriminate between two'state and downhill folding. J. Am. Chem. Soc. 2004, 126, 8596-8597.

(9) Naganathan, A. N.; Muñoz, V. Thermodynamics of Downhill Folding: Multi-Probe Analysis of PDD, a Protein that Folds Over a Marginal Free Energy Barrier. J. Phys. Chem. B 2014, 118, 8982-8994.

(10) Muñoz, V.; Campos, L. A.; Sadqi, M. Limited cooperativity in protein folding. Curr. Opin. Struct. Biol. 2016, 36, 58-66.

(11) Narayan, A.; Campos, L. A.; Bhatia, S.; Fushman, D.; Naganathan, A. N. Graded structural polymorphism in a bacterial thermosensor protein. J. Am. Chem. Soc. 2017, 139, 792-802.

(12) Wako, H.; Saito, N. Statistical Mechanical Theory of Protein Conformation 0.2. Folding Pathway for Protein. J. Phys. Soc. Jpn. 1978, 44, 1939-1945.

(13) Muñoz, V.; Eaton, W. A. A simple model for calculating the kinetics of protein folding from three-dimensional structures. Proc. Natl. Acad. Sci. U. S. A. 1999, 96, 11311-11316.

(14) Naganathan, A. N. Predictions from an Ising-like Statistical Mechanical Model on the Dynamic and Thermodynamic Effects of Protein Surface Electrostatics. J. Chem. Theory Comput. 2012, 8, 46464656.

(15) Myers, J. K.; Pace, C. N.; Scholtz, J. M. Denaturant m-Values and Heat-Capacity Changes - Relation to Changes in Accessible Surface-Areas of Protein Unfolding. Protein Sci. 1995, 4, 2138-2148.

(16) Naganathan, A. N.; Sanchez-Ruiz, J. M.; Munshi, S.; Suresh, S. Are Protein Folding Intermediates the Evolutionary Consequence of Functional Constraints? J. Phys. Chem. B 2015, 119, 1323-1333.

(17) Strickland, E. H.; Beychok, S. Aromatic contributions to circular dichroism spectra of proteins. Crit. Rev. Biochem. 1974, 2, 113-175.

(18) Chakrabartty, A.; Kortemme, T.; Padmanabhan, S.; Baldwin, R. L. Aromatic side-chain contribution to far-ultraviolet circular dichroism of helical peptides and its effect on measurement of helix propensities. Biochemistry 1993, 32, 5560-5565.

(19) Schuler, B.; Soranno, A.; Hofmann, H.; Nettels, D. SingleMolecule FRET Spectroscopy and the Polymer Physics of Unfolded and Intrinsically Disordered Proteins. Annu. Rev. Biophys. 2016, 45, 207-231.

(20) Uversky, V. N. Size-exclusion chromatography in structural analysis of intrinsically disordered proteins. Methods Mol. Biol. 2012, 896, 179-194.

(21) Cerminara, M.; Desai, T. M.; Sadqi, M.; Muñoz, V. Downhill protein folding modules as scaffolds for broad-range ultrafast biosensors. J. Am. Chem. Soc. 2012, 134, 8010-8013.

(22) Naganathan, A. N.; Orozco, M. The Native Ensemble and Folding of a Protein Molten-Globule: Functional Consequence of Downhill Folding. J. Am. Chem. Soc. 2011, 133, 12154-12161.

(23) Madrid, C.; Nieto, J. M.; Juarez, A. Role of the Hha/YmoA family of proteins in the thermoregulation of the expression of virulence factors. Int. J. Med. Microbiol. 2001, 291, 425-432.

(24) Bae, S.-H.; Liu, D.; Lim, H. M.; Lee, Y.; Choi, B.-S. Structure of the Nucleoid-Associated Protein Cnu Reveals Common Binding Sites for H-NS in Cnu and Hha. Biochemistry 2008, 47, 1993-2001.

(25) Cordeiro, T. N.; Garcia, J.; Bernado, P.; Millet, O.; Pons, M. A Three-protein Charge Zipper Stabilizes a Complex Modulating Bacterial Gene Silencing. J. Biol. Chem. 2015, 290, 21200-21212.

(26) Cooper, A. Thermodynamic Fluctuations in Protein Molecules. Proc. Natl. Acad. Sci. U. S. A. 1976, 73, 2740-2741.

(27) Honeycutt, J. D.; Thirumalai, D. Metastability of the folded states of globular proteins. Proc. Natl. Acad. Sci. U. S. A. 1990, 87, $3526-3529$.

(28) Jha, S. K.; Dhar, D.; Krishnamoorthy, G.; Udgaonkar, J. B. Continuous dissolution of structure during the unfolding of a small protein. Proc. Natl. Acad. Sci. U. S. A. 2009, 106, 11113-11118.

(29) Campos, L. A.; Sadqi, M.; Liu, J. W.; Wang, X.; English, D. S.; Muñoz, V. Gradual Disordering of the Native State on a Slow Two-
State Folding Protein Monitored by Single-Molecule Fluorescence Spectroscopy and NMR. J. Phys. Chem. B 2013, 117, 13120-13131.

(30) Law, A. B.; Sapienza, P. J.; Zhang, J.; Zuo, X.; Petit, C. M. Native State Volume Fluctuations in Proteins as a Mechanism for Dynamic Allostery. J. Am. Chem. Soc. 2017, 139, 3599. 